\title{
Development of a multivariate model to predict significant coronary artery disease in Thai patients with left ventricular systolic dysfunction and determine the applicability of coronary angiography: a single-center, retrospective, case-control study
}

\author{
Ratikorn Methavigul*, Komsing Methavigul
}

\section{Abstract}

Background: Coronary angiography $(\mathrm{CAG})$ or stress imaging has been performed in almost all Thai patients with left ventricular (LV) systolic dysfunction. If CAG results reveal insignificant coronary stenosis, such patients are diagnosed with nonischemic cardiomyopathy (NICM); however, CAG is considered to provide no benefit and may even harm these patients because it is invasive.

Objectives: To identify predictors associated with significant coronary artery disease (CAD) (stenosis) in Thai patients with LV systolic dysfunction without angina and without LV regional wall motion abnormality and create a prediction score.

Method: Retrospective data from patients at a single tertiary-care center with LV systolic dysfunction (LV ejection fraction $<50 \%$ ) diagnosed between August 2000 and October 2014 were separated into a group with ischemic cardiomyopathy (ICM) and a group with NICM according to CAG. Predictors associated with CAD found in normal populations were determined. Multivariate analysis was used to identify predictors associated with significant coronary stenosis in patients with LV systolic dysfunction to develop a model to create a prediction score.

Results: We included data registered from 240 Thai patients with LV systolic dysfunction. Predictors associated with ICM were age ( $>60$ years), sex (male), and a history of diabetes mellitus (DM). Predictors associated with NICM were body mass index (BMI) $>25 \mathrm{~kg} / \mathrm{m}^{2}$ and the presence of left bundle branch block (LBBB) on electrocardiography. A simplified equation to predict significant CAD in patients with LV systolic dysfunction is: $3($ male sex $)+3($ age $>60 \mathrm{y})-$ $5\left(\mathrm{BMI}>25 \mathrm{~kg} / \mathrm{m}^{2}\right)-5(\mathrm{LBBB})+5(\mathrm{DM})-5$. The sensitivity and specificity of this score are $60.5 \%$ and $85.1 \%$, respectively.

Conclusion: Our prediction score has modest sensitivity, but high specificity for predicting significant CAD and can be used to determine who should not undergo CAG.

Keywords: coronary artery disease, ischemic cardiomyopathy, LV systolic dysfunction, nonischemic cardiomyopathy, predictors

*Correspondence to: Ratikorn Methavigul, Department of Cardiology, Central Chest Institute of Thailand, Nonthaburi 11000, Thailand,

e-mail: ratikorn@ccit.mail.go.th

Department of Cardiology, Central Chest Institute of Thailand, Nonthaburi 11000, Thailand

כ Open Access. ๑ 2017 Ratikorn Methavigul, Komsing Methavigul published by Sciendo. (c) BY-NC-ND This work is licensed under the Creative Commons Attribution NonCommercial-NoDerivatives 4.0 License. 
Left ventricular (LV) systolic dysfunction is the consequence of many etiologies including dilated cardiomyopathy, alcoholic cardiomyopathy, peripartum cardiomyopathy, tachycardia-induced cardiomyopathy, and ischemic cardiomyopathy (ICM) [1-4]. Coronary artery disease (CAD) is the most common treatable cause of LV systolic dysfunction, and revascularization has been shown to improve LV function in this group of patients [5-7].

Currently, there is no clear recommendation for whether patients with LV systolic dysfunction should undergo coronary angiography (CAG). Thus, in many institutions, CAG or stress imaging has been conducted in all patients suffering from LV systolic dysfunction. Patients can be divided into 2 groups based on CAG results: a group with ICM (significant coronary artery stenosis) and a group with nonischemic cardiomyopathy (NICM) (normal CAG or insignificant stenosis). CAG is considered to have no benefit (and may harm) for those in the NICM group because it is invasive. Moreover, CAG and stress imaging, such as vasodilator stress MRI, are of high cost and can be performed only in experienced centers, while dobutamine stress echocardiography is an operator-dependent procedure, and its sensitivity and specificity may vary among operators.

In previous guidelines $[8,9]$, there are many risk scores that can predict future cardiovascular disease in the general population including Systematic Coronary Risk Evaluation Project (SCORE) from the European Society of Cardiology (ESC) [8] and the pooled cohort equation for atherosclerotic cardiovascular disease (ASCVD) risk from the American College of Cardiology (ACC) and American Heart Association (AHA) [9]. However, there is no standard recommendation for predicting significant CAD (stenosis) in patients with LV systolic dysfunction.

Following previous studies, Whellan et al. [10] and Wongchareon et al. [11] have proposed a predictive model to differentiate ICM from NICM. Both studies recruited patients with LV systolic dysfunction whether or not they had chest pain or a LV regional wall motion abnormality seen by echocardiography. These 2 predictors were found more in the group with ICM than in that with NICM. However, in our clinical practice, patients with LV systolic dysfunction who have chest pain or LV regional wall motion abnormalities almost always undergo CAG because of the high likelihood of significant CAD in these patients. Therefore, the results of these 2 studies cannot be applied to patients without chest pain or LV regional wall motion abnormality.

The aims of the present study were to identify predictors associated with significant coronary stenosis in Thai patients with LV systolic dysfunction without angina and without LV regional wall motion abnormality and develop using multiple logistic regression (backward stepwise method) a simple and reliable model to create a score to predict CAD from multiple significant predictors. This score could be used as a diagnostic screening tool to predict the possibility of significant coronary artery disease in patients with LV systolic dysfunction without angina and without LV regional wall motion abnormality.

\section{Materials and methods}

After approval of the study protocol by the Central Chest Institute of Thailand Institutional Review Board (approval No. 103/2557), we conducted a retrospective, observational, single-center, case-control study of Thai adult (at least 18 years old) patients with LV systolic dysfunction in our tertiary-care hospital. Data registered at the Central Chest Institute of Thailand from 240 patients with LV systolic dysfunction diagnosed between August 2000 and October 2014 were included. The study included data from patients with LV ejection fraction (LVEF) $<50 \%$. Data from patients were excluded if the patient had a history of CAD (stable CAD or acute coronary syndrome), history of revascularization (percutaneous coronary intervention (PCI) or coronary artery bypass grafting (CABG)), history of angina, regional wall motion abnormality from echocardiography, contraindication to antiplatelet therapy, more than a moderate degree of valvular heart disease [12] except mitral regurgitation from annular dilatation, history of current or former use of addictive drugs or substance use (e.g. amphetamines or cocaine), and stress cardiomyopathy, and if patients who refused CAG.

Data from patients were divided into 2 groups according to CAG results. These 2 groups were the group with ICM and the group with NICM. Predictor variables associated with CAD were collected. The outcome variables were the predictors that were found to be significantly associated with CAD in previous studies [1].

LV systolic dysfunction is defined as LVEF $<50 \%$. The definition of ICM has been modified from previous 2012 American College of Cardiology Foundation (ACCF)/AHA Guidelines for the Diagnosis and Management of Patients with Stable Ischemic Heart Disease [13] and Felker et al. [1], which included patients with LV systolic dysfunction and $\geq 50 \%$ stenosis of left main or $\geq 70 \%$ stenosis of proximal left anterior descending artery (LAD) or patients with $\geq 70 \%$ stenosis of 2 or more epicardial vessels (LAD, left circumflex artery, or right coronary artery).

Predictors of significant CAD that were included in the present study are age ( $>60$ years at diagnosis); sex (male); smoking habit within 2 years of diagnosis; history of diabetes 
mellitus (DM; fasting blood sugar >126 mg/dL); hypertension (systolic blood pressure $>140 \mathrm{mmHg}$, diastolic blood pressure $>90 \mathrm{mmHg}$, or taking an antihypertensive drug); dyslipidemia (low-density lipoprotein (LDL) $>160 \mathrm{mg} / \mathrm{dL}$ or triglyceride $>150 \mathrm{mg} / \mathrm{dL}$ plus high-density lipoprotein (HDL) $<40 \mathrm{mg} / \mathrm{dL}$ in men or HDL $<50 \mathrm{mg} / \mathrm{dL}$ in women); family history of premature $\mathrm{CAD}$ (male relative $<55$ years old, female relative $<65$ years old); history of other atherosclerotic disease, such as peripheral arterial disease (ankle-brachial index $<0.9$ or known peripheral artery disease); ischemic stroke; pathologic Q wave in contiguous leads in any ECG; and body mass index (BMI) at the diagnosis $>25 \mathrm{~kg} / \mathrm{m}^{2}$. Only available data were analyzed. All predictors are certain CAD risk factors from previous study [1].

The literature indicates that the overall prevalence of the predictors described earlier in the NICM group are 55\% for age, $54 \%$ for sex (male), $56.6 \%$ for hypertension, $51 \%$ for DM, 20.8\% for hyperlipidemia, and 51\% for smoking [1]. We chose prevalence of hypertension $\left(P_{2}=56.6 \%\right)$ for sample size calculation. We specified 0.05 for type I error, 0.20 for type II error with $80 \%$ power, and an odds ratio of 2 .

$P_{2}=$ NICM group predictor exposure rate

$P_{1}=$ ICM group predictor exposure rate

From the formula:

$$
P_{1}=\frac{(\mathrm{OR}) P_{2}}{(\mathrm{OR}) P_{2}+\left(1-P_{2}\right)}
$$

The sample size $(N)$ was calculated using the following formula:

$$
\begin{aligned}
N & =\frac{\left\{Z_{1-\alpha / 2} \sqrt{2 P_{2}\left(1-P_{2}\right)}+Z_{1-\beta} \sqrt{P_{1}\left(1-P_{1}\right)+P_{2}\left(1-P_{2}\right)}\right\}^{2}}{\left(P_{1}-P_{2}\right)^{2}} \\
& =\frac{\{1.96 \sqrt{(2 \times 0.566)(0.434)}+0.842 \sqrt{0.723(0.277)+0.566(0.434)}\}^{2}}{(0.157)^{2}}
\end{aligned}
$$

The calculated sample size was data from 73 patients for each group. We increased the sample size by $10 \%$ to data from 80 patients for each group in case of missing or incomplete data and set the ratio of ICM to NICM as 1:2. Thus, overall sample size for this study was data from 240 patients (80 patients in the ICM group and 160 patients in the NICM group).

We used PASW Statistics for Windows, version 18.0 (SPSS) for all our analyses. Characteristics of patients from whom data were included are summarized using descriptive statistics, including frequencies and percentages for categorical variables. Continuous variables are reported as mean values, normally distributed variables as standard deviation, and non-normally distributed variables as median, minimum, and maximum. The normality of distribution of the variables was examined using a Kolmogorov-Smirnov test. Differences between normally distributed variables were investigated using a Student $t$ test to compare mean values, and a $\chi^{2}$ test was used to compare proportions between 2 groups.

Variables found significantly associated with and affected by CAD in multivariate logistic regression were selected predictors with a base of $P<0.2$ in univariate logistic regression, and a backward stepwise selection technique was used to find the best model. Interaction effect between variables was assessed using a Mantel-Hanzel test of homogeneity. Multicollinearity between independent variables was assessed using variance inflation factors (VIFs) before interpreting the final output. For all tests performed, a 2-tailed test result with $P<0.05$ was considered as significant. The best model was multiple logistic regression (backward stepwise method).

The significant predictors of CAD were used to calculate CAD risk score. To validate the predictive logistic model, we generated a random sample from the original dataset for a larger database. The size of sample was drawn from the prevalence of CAD and sampling data without replacement. The cutoff score was considered the highest predictive accuracy of CAD for the model.

The cutoff score considered to have the highest predictive accuracy for CAD was identified using a receiver operating characteristic (ROC) curve and presented as sensitivity, specificity, positive predictive value (PPV), negative predictive value (NPV), likelihood ratio (LR), and accuracy. For all tests performed, a 2 -tailed $P<0.05$ was considered as significant.

\section{Results}

We included data from 240 patients, those with LV systolic dysfunction and who underwent CAG at our tertiary-care center from August 2000 to October 2014. CAG showed significant coronary artery stenosis in 80 patients and normal or nonsignificant stenosis in 160 patients. The mean age of patients was $55.7 \pm 12.2$ years. Male patients accounted for $70 \%, 53 \%$ of patients had hypertension, and $48 \%$ of patients had dyslipidemia. One-third of patients had DM, and $56 \%$ had smoked in the previous 2 years or were current smokers at the time of diagnosis. Baseline LVEF was $26.7 \pm 8.6 \%$, and LVEF at follow-up visit was $40.4 \pm 13.7 \%$. The time between the original and the follow-up LVEF was $755 \pm 788$ days (range, 6 to 3,782 days). Baseline characteristics are summarized in Table $\mathbf{1 .}$

An initial univariate analysis was conducted. Predictors that were significantly high in those with $\operatorname{ICM}(P<0.05)$ 
Table 1. Baseline characteristics of the patients with left ventricular systolic dysfunction

\begin{tabular}{|c|c|c|c|c|}
\hline \multirow[b]{2}{*}{ Characteristics } & \multicolumn{3}{|c|}{ Number $(\%)$ or mean \pm SD } & \\
\hline & $\begin{array}{r}\text { Total } \\
(\mathrm{N}=\mathbf{2 4 0})\end{array}$ & $\begin{array}{r}\text { Ischemic cardiomyopathy } \\
\qquad(\mathbf{n}=\mathbf{8 0})\end{array}$ & $\begin{array}{r}\text { Nonischemic cardiomyopathy } \\
\qquad(n=160)\end{array}$ & \\
\hline Male sex & $167(70)$ & 61 (76) & $106(66)$ & 0.11 \\
\hline Age (years) & $55.7 \pm 12.2$ & $60.3 \pm 10.6$ & $53.5 \pm 12.4$ & $<0.001$ \\
\hline$\leq 60$ & $157(73)$ & $41(51)$ & $116(73)$ & 0.001 \\
\hline Body weight (kg) & $65.5 \pm 16.8$ & $58.7 \pm 11.8$ & $69.0 \pm 17.9$ & $<0.001$ \\
\hline Height $(\mathrm{cm})$ & $163.4 \pm 8.6$ & $163.2 \pm 7.0$ & $163.5 \pm 9.5$ & 0.77 \\
\hline Body mass index $\left(\mathrm{kg} / \mathrm{m}^{2}\right)$ & $24.4 \pm 5.2$ & $22.0 \pm 3.8$ & $25.6 \pm 5.3$ & $<0.001$ \\
\hline$\leq 25$ & $142(59.7)$ & $63(79.7)$ & 79 (49.7) & $<0.001$ \\
\hline Creatinine (mg/dL) & $1.1 \pm 0.2$ & $1.1 \pm 0.2$ & $1.1 \pm 0.3$ & 0.54 \\
\hline Baseline LVEF (\%) & $26.7 \pm 8.6$ & $26.0 \pm 9.3$ & $27.0 \pm 8.3$ & 0.41 \\
\hline LVEF at follow-up visit (\%) & $40.4 \pm 13.7$ & $37.2 \pm 13.6$ & $42.2 \pm 37.2$ & 0.06 \\
\hline \multicolumn{5}{|l|}{ LVEF improvement } \\
\hline No & $103(43)$ & $40(50)$ & $63(39)$ & 0.23 \\
\hline Yes & $33(14)$ & $8(10)$ & $25(16)$ & \\
\hline N/A & $104(43)$ & $32(40)$ & $72(45)$ & \\
\hline \multicolumn{5}{|l|}{ ECG } \\
\hline Pathologic Q wave in contiguous leads & $24(10)$ & $14(18)$ & $10(6)$ & 0.006 \\
\hline LBBB & $15(6)$ & $2(3)$ & $13(8)$ & 0.09 \\
\hline Atrial fibrillation or atrial flutter & $42(18)$ & $8(10)$ & $34(21)$ & 0.03 \\
\hline \multicolumn{5}{|l|}{ Underlying diseases } \\
\hline Diabetes mellitus & $72(30)$ & $39(49)$ & $33(21)$ & $<0.001$ \\
\hline Hypertension & $127(53)$ & $44(55)$ & $83(52)$ & 0.65 \\
\hline Dyslipidemia & $114(48)$ & $49(61)$ & $65(41)$ & 0.003 \\
\hline Obstructive sleep apnea & $3(1)$ & $0(0)$ & $3(2)$ & 0.55 \\
\hline LV thrombus & $11(5)$ & $3(4)$ & $8(5)$ & 0.76 \\
\hline Atrial fibrillation or atrial flutter & $42(18)$ & $8(10)$ & $34(21)$ & 0.03 \\
\hline Valvular heart disease & $80(33)$ & $30(38)$ & $50(31)$ & 0.33 \\
\hline Chronic kidney disease & $7(3)$ & $3(4)$ & $4(3)$ & 0.69 \\
\hline Peripheral arterial disease & $3(1)$ & $3(4)$ & $0(0)$ & 0.04 \\
\hline Pulmonary hypertension & $22(9)$ & $13(16)$ & $9(6)$ & 0.007 \\
\hline Transient ischemic attack & $3(1)$ & $1(1)$ & $2(1)$ & $>0.99$ \\
\hline Past history of stroke & $6(3)$ & $1(1)$ & $5(3)$ & 0.67 \\
\hline COPD & $19(8)$ & $12(15)$ & $7(4)$ & 0.004 \\
\hline Smoking habit & $134(56)$ & $50(63)$ & $84(53)$ & 0.14 \\
\hline Alcohol habit & $94(39)$ & $27(34)$ & $67(42)$ & 0.22 \\
\hline Family history of premature coronary artery disease & $44(18)$ & $12(15)$ & $32(20)$ & 0.35 \\
\hline
\end{tabular}

COPD, chronic obstructive pulmonary disease; ECG, electrocardiogram; LBBB, left bundle branch block; LVEF, left ventricular ejection fraction; N/A, not available; SD, standard deviation

were age ( $>60$ years), pathologic Q wave in contiguous leads, $\mathrm{DM}$, and dyslipidemia. Predictors that were significantly high among those in the non-ICM group were BMI $>25 \mathrm{~kg} / \mathrm{m}^{2}$ and atrial fibrillation. The test of homogeneity showed no interaction between variables. The VIF showed no evidence of a multicollinearity problem among independent variables. After the multivariate analysis, 5 predictors were identified to be significant for discrimination. Predictors that were found associated with increased probability of ICM (risk factors) were age $(>60$ years old), sex (male), and DM $(P<0.05)$. Predictors that were found associated with increased probability of insignificant coronary artery stenosis (protective factors) were BMI $>25 \mathrm{~kg} / \mathrm{m}^{2}$ and left bundle branch block (LBBB) on ECG $(P<0.05)$ as shown in Table 2.

Based on these 5 predictors, we can derive the original discriminating equation and the simplified version to predict the possibility of significant CAD in patients with LV systolic dysfunction as shown in Table 3.

In the present study, we found that the suitable cutoff is 0 (Figure 1, area under the curve $=80.13 \%(95 \%$ confidence 
Table 2. Predictors of ischemic cardiomyopathy

\begin{tabular}{|c|c|c|c|c|}
\hline \multirow[b]{2}{*}{ Predictors } & \multicolumn{2}{|c|}{ Univariate analysis } & \multicolumn{2}{|c|}{ Multivariate analysis } \\
\hline & $\begin{array}{r}\text { OR } \\
(95 \% \mathrm{Cl} \text { of } \mathrm{OR})\end{array}$ & $P$ & $\begin{array}{r}\text { OR } \\
(95 \% \mathrm{Cl} \text { of OR) }\end{array}$ & $P$ \\
\hline Male sex & $1.64(0.89-3.01)$ & 0.11 & $2.55(1.23-5.30)$ & 0.012 \\
\hline Age (>60 years) & $2.51(1.43-4.39)$ & 0.001 & $2.94(1.52-5.69)$ & 0.001 \\
\hline $\mathrm{BMI}\left(>25 \mathrm{~kg} / \mathrm{m}^{2}\right)$ & $0.84(0.78-0.90)$ & $<0.001$ & $0.21(0.10-0.42)$ & $<0.001$ \\
\hline $\begin{array}{l}\text { LVEF at follow-up } \\
\text { visit (\%) }\end{array}$ & $0.97(0.95-1.0)$ & 0.06 & - & - \\
\hline $\begin{array}{l}\text { Pathologic } \\
\text { Q wave }\end{array}$ & $3.18(1.34-7.53)$ & 0.008 & - & - \\
\hline LBBB & $0.29(0.06-1.32)$ & 0.11 & $0.18(0.03-0.98)$ & 0.047 \\
\hline Diabetes mellitus & $3.66(2.05-6.55)$ & $<0.001$ & $5.93(2.94-11.98)$ & $<0.001$ \\
\hline Dyslipidemia & $2.31(1.33-4.0)$ & 0.003 & - & - \\
\hline $\begin{array}{l}\text { Atrial fibrillation } \\
\text { or atrial flutter }\end{array}$ & $0.41(0.18-0.94)$ & 0.04 & - & - \\
\hline $\begin{array}{l}\text { Pulmonary } \\
\text { hypertension }\end{array}$ & $3.26(1.33-7.99)$ & 0.01 & - & - \\
\hline COPD & $3.86(1.46-10.23)$ & 0.007 & - & - \\
\hline Smoking habit & $1.51(0.87-2.61)$ & 0.14 & - & - \\
\hline
\end{tabular}

$\mathrm{BMI}$, body mass index; $\mathrm{Cl}$, confidence interval; $\mathrm{COPD}$, chronic obstructive pulmonary disease; $L B B B$, left bundle branch block; LVEF, left ventricular ejection fraction; $\mathrm{OR}$, odds ratio

Table 3. Coronary artery disease prediction score for left ventricular systolic dysfunction

\section{Original equation}

CAD score $=0.937$ (male sex) +1.080 (age $>60$ years) -1.572 (BMI $>$ $\left.25 \mathrm{~kg} / \mathrm{m}^{2}\right)-1.720($ LBBB $)+1.780(\mathrm{DM})-1.749$

\section{Simplified equation ${ }^{+}$}

CAD score $=3($ male sex $)+3$ (age $>60$ years $)-5\left(\right.$ BMI $\left.>25 \mathrm{~kg} / \mathrm{m}^{2}\right)-$ $5(\mathrm{LBBB})+5(\mathrm{DM})-5$

Sex: male $=1$, female $=0$

Age: $>60$ years old $=1, \leq 60$ years old $=0$

BMI: $>25 \mathrm{~kg} / \mathrm{m}^{2}=1, \leq 25 \mathrm{~kg} / \mathrm{m}^{2}=0$

LBBB: yes $=1$, no $=0$

DM: yes $=1$, no $=0$

${ }^{\dagger}$ Multiplying by 3 and then rounding the coefficients

$B M I$, body mass index; $C A D$, coronary artery disease; DM, diabetes mellitus; LBBB, left bundle branch block

interval (CI) $79.35 \%-80.92 \%)$ ). Therefore, there is a possibility to find significant coronary artery stenosis if the patient's calculated CAD score is $\geq 0$. The sensitivity and specificity of this score are $60.5 \%(95 \%$ CI $58.9 \%-62.0 \%)$ and $85.1 \%(95 \%$ CI $84.3 \%-85.9 \%)$, respectively, with a PPV $66.8 \%(95 \%$ CI 65.3\%-68.4\%), NPV 81.3\% (95\% CI 80.1\%-82.1\%), LR+ 4.06 (95\% CI 3.83-4.30), LR- 0.46 (95\% CI 0.45-0.48), and overall accuracy $76.93 \%$ (Table 4 ).

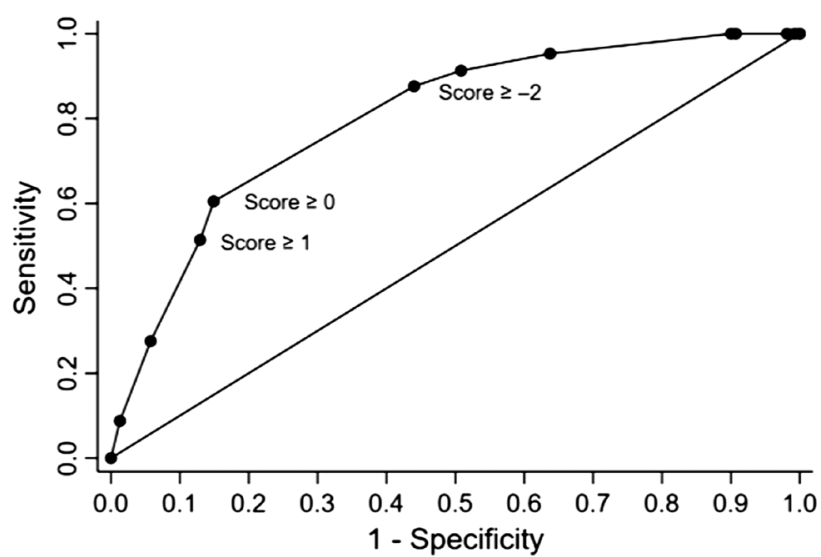

Area under ROC curve $=0.8013$

Figure 1. Receiver operating characteristic curve to predict significant coronary artery disease in patients with left ventricular systolic dysfunction

ROC, receiver operating characteristic

\section{Discussion}

We sought to identify predictors associated with significant CAD (stenosis) in patients with LV systolic dysfunction and create a simple equation to differentiate patients with ICM from those with NICM. We can use this classification to determine which patients would benefit from CAG. Predictors for ICM were age ( $>60$ years), sex (male), and DM. Predictors for NICM were BMI $>25 \mathrm{~kg} / \mathrm{m}^{2}$ and LBBB on ECG.

Well-known risk factors for CAD are older age, smoking, $\mathrm{DM}$, hypertension, dyslipidemia, and family history of premature atherosclerotic disease [14, 15]. Advanced age is an important risk for CAD in both men and women, especially in patients aged $>60$ years [16]. For patients with LV systolic dysfunction, the mean age of patients with ICM (64 years) was significantly more than that of patients with NICM (55 years) [1]. We also found an association between increasing age and CAD. A similar association was found with DM, which is also associated with significant CAD.

From the European Guidelines for Cardiovascular Disease Prevention in Clinical Practice [8] that uses SCORE to determine risks for cardiovascular disease in the general population, the risk factor includes sex and the pooled cohort equation for ASCVD risk score from the ACC/AHA Guidelines on the Assessment of Cardiovascular Risk [9]. We also found a distinction between sexes in the present study. We found that male patients with LV systolic dysfunction have more significant CAD than female patients.

In the present study, patients with BMI $>25 \mathrm{~kg} / \mathrm{m}^{2}$ were more likely to have NICM than ICM. This can be explained 
Table 4. Sensitivity, specificity, positive predictive value, negative predictive value, and accuracy for each cutoff point

\begin{tabular}{lrrrrr}
\hline Cutoff score & Sensitivity, \% (95\% Cl) & Specificity, \% (95\% Cl) & PPV, \% (95\% Cl) & NPV, \% (95\% Cl) & Accuracy (\%) \\
\hline-2 & $87.6(86.5-88.6)$ & $56.0(54.9-57.1)$ & $49.7(48.5-50.9)$ & $90.1(89.2-90.9)$ & 66.48 \\
0 & $60.5(58.9-62.0)$ & $85.1(84.3-85.9)$ & $66.8(65.3-68.4)$ & $81.3(80.4-82.1)$ & 76.93 \\
1 & $51.4(49.8-53.0)$ & $87.1(86.3-87.8)$ & $66.4(64.6-68.0)$ & $78.3(77.4-79.2)$ & 75.23 \\
\hline
\end{tabular}

$\mathrm{Cl}$, confidence interval; NPV, negative predictive value; PPV, positive predictive value

by data from previous studies, which indicate that although the best survival in the general population is in those with BMI $20-24.9 \mathrm{~kg} / \mathrm{m}^{2}$, for patients with chronic diseases, such as chronic kidney disease and chronic heart failure, overweight and obese patients have better survival rate. This phenomenon, known as the obesity paradox [17], was first described by Gruberg et al. [18]. They found that in patients with CAD undergoing PCI, the risk of in-hospital complications and cardiac death in obese patients is less than that in patients with normal BMI. Moreover, further study reveals that the risk of adverse events in patients with chronic heart failure is highest in patients with low BMI $\left(<20 \mathrm{~kg} / \mathrm{m}^{2}\right)$ and the risk for cardiovascular mortality and hospitalization was lowest in patients who were overweight (BMI 25 to $29.9 \mathrm{~kg} / \mathrm{m}^{2}$ ) [19]. The reason for the obesity paradox is unclear. There are 2 possible explanations. The first is that obese patients tend to receive guideline-based optimal medical therapy (i.e. statins, angiotensin-converting enzyme inhibitors, and $\beta$-blockers) more than nonobese patients. The second explanation is that the physicians tend to advise obese patients about lifestyle modifications, such as to quit smoking, eat a suitable diet, and exercise, more than those with normal BMI [20].

LBBB occurs when there are conduction defects at both the left anterior and left posterior fascicles. Normally, the left bundle branch is supplied by many branches of the coronary artery. Therefore, if a complete $\mathrm{LBBB}$ as a result of CAD occurs, patients must have large area of ischemia and have more than 1 coronary artery affected [21]. Thus, in those with LV systolic dysfunction, complete LBBB is usually a result of myocardial disease than CAD.

We found no significant differences in the prevalence of hypertension and dyslipidemia in the present study. The most likely explanation is that most patients with preexisting hypertension and dyslipidemia had received antihypertensive drugs and statins and advised to modify their lifestyle. Therefore, blood pressure and lipid level are well controlled, reducing cardiovascular risk. As seen from the calculation of 10-year risk of cardiovascular disease in SCORE from ESC and pooled cohort equation for ASCVD risk score from ACC/AHA $[8,9]$, predictors are systolic blood pressure and total cholesterol,
HDL, or LDL levels. If the patients have been treated, blood pressure and lipid profile will decrease and cardiovascular risk will be reduced.

The limitations of our study include its retrospective and single-center design. Certain data regarding family history of premature cardiovascular disease and smoking were limited, but these were similar between the 2 groups. Another limitation is the method of internal validation, in that we used a random sample generated from the original data set. External validation with another data set was not conducted because of the limited population that met the inclusion criteria (all patients who have chest pain or LV regional wall motion abnormalities were excluded). The 240 patients in this study were all patients with LV systolic dysfunction who met the inclusion criteria diagnosed between August 2000 and October 2014 (14 years) in our center. Before 2000, the data were limited. An alternative sampling method such as a bootstrap validation approach by sampling individuals with replacement from the original sample might have been better. External validity is needed by having another set of samples independent of the sample from which the predictive model was derived. A further prospective multicenter study to validate the prediction score is warranted.

Because of the high specificity and NPV, this equation is suitable for excluding patients with NICM who should not undergo CAG or stress imaging. The score generated can be used to determine whether or not CAG should be performed. Patients with LV systolic dysfunction and a score $<0$ are not likely to benefit from CAG because there is little chance that it will detect significant CAD.

\section{Conclusion}

In patients with LV systolic dysfunction, predictors associated with ICM are age ( $>60$ years), sex (male), and DM. Predictors associated with NICM are BMI $>25 \mathrm{~kg} / \mathrm{m}^{2}$ and LBBB on ECG. The prediction score has modest sensitivity, but high specificity for predicting significant coronary stenosis and can be used to determine who will not benefit from CAG. However, further prospective multicenter study to validate the prediction score is warranted. 
Author contributions. RM and KM contributed to the conception and design of the study and analyzed and interpreted the data collected substantially by RM who drafted the manuscript. Both authors critically revised the manuscript, approved the final version submitted for publication, and take responsibility for the statements made therein.

Acknowledgments. This research did not receive any specific grant from funding agencies in the public, commercial, or notfor-profit sectors.

Conflict of interest statement. The authors have each completed and submitted an ICMJE Uniform Disclosure Form for Potential Conflicts of Interest. Neither of the authors disclose any potential conflict of interest.

\section{References}

[1] Felker GM, Shaw LK, O'Connor CM. A standardized definition of ischemic cardiomyopathy for use in clinical research. J Am Coll Cardiol. 2002; 39:210-8.

[2] Sisakian H. Cardiomyopathies: evolution of pathogenesis concepts and potential for new therapies. World J Cardiol. 2014; 6:478-94.

[3] Malcom J, Arnold O, Howlett JG, Ducharme A, Ezekowitz JA, Gardner M, et al. Canadian Cardiovascular Society Consensus Conference Guidelines on Heart Failure 2008 update: best practices for the transition of care of heart failure patients, and the recognition, investigation and treatment of cardiomyopathies. Can J Cardiol. 2008; 24:21-40.

[4] Wexler RK, Elton T, Pleister A, Feldman D. Cardiomyopathy: an overview. Am Fam Physician. 2009; 79:778-84.

[5] Pinto N, Haluska B, Mundy J, Griffin R, Wood A, Shah P. Ischemic cardiomyopathy: midterm survival and its predictors. Asian Cardiovasc Thorac Ann. 2012; 20:669-74.

[6] Panza JA, Velazquez EJ, She L, Smith PK, Nicolau JC, Favaloro RR, et al. Extent of coronary and myocardial disease and benefit from surgical revascularization in ischemic LV dysfunction. J Am Coll Cardiol. 2014; 64:553-61. Erratum in J Am Coll Cardiol. 2014; 64:1539.

[7] Romero-Farina G, Candell-Riera J, Aguadé-Bruix S, Castell-Conesa J, de Léon G, Igual A. Predictors of improved left ventricular systolic function after surgical revascularization in patients with ischemic cardiomyopathy. Rev Esp Cardiol. 2007; 60:943-51.

[8] Perk J, De Backer G, Gohlke H, Graham I, Reiner Z, Verschuren WM, et al. European Guidelines on Cardiovascular Disease Prevention in Clinical Practice (version 2012). The Fifth Joint Task Force of the European Society of Cardiology and other societies on cardiovascular disease prevention in clinical practice (constituted by representatives of nine societies and by invited experts). Int J Behav Med. 2012; 19:403-88.

[9] Goff DC Jr, Lloyd-Jones DM, Bennett G, Coady S, D’Agostino RB Sr, Gibbons R, et al. 2013 ACC/AHA guideline on the assessment of cardiovascular risk: a report of the American College of Cardiology/ American Heart Association Task Force on Practice Guidelines. J Am Coll Cardiol. 2014; 63:2935-59.
[10] Whellan DJ, Tuttle RH, Velazquez EJ, Shaw LK, Jollis JG, Ellis W, et al. Predicting significant coronary artery disease in patients with left ventricular dysfunction. Am Heart J. 2006; 152:340-7.

[11] Wongchareon W, Phrommintikul A, Kanjanavanit R, Kuanprasert S, Sukonthasarn A. A predictive model for distinguishing ischemic from non-ischemic cardiomyopathy. J Med Assoc Thai. 2005; 88:1689-96

[12] Bonow RO, Carabello BA, Chatterjee K, de Leon AC Jr, Faxon DP, Freed MD, et al. 2008 focused update incorporated into the ACC/AHA 2006 guidelines for the management of patients with valvular heart disease: a report of the American College of Cardiology/American Heart Association Task Force on Practice Guidelines (Writing Committee to revise the 1998 guidelines for the management of patients with valvular heart disease). Endorsed by the Society of Cardiovascular Anesthesiologists, Society for Cardiovascular Angiography and Interventions, and Society of Thoracic Surgeons. J Am Coll Cardiol. 2008; 52:e1-142.

[13] Fihn SD, Gardin JM, Abrams J, Berra K, Blankenship JC, Dallas AP, et al. 2012 ACCF/AHA/ACP/AATS/PCNA/SCAI/STS Guideline for the diagnosis and management of patients with stable ischemic heart disease: a report of the American College of Cardiology Foundation/American Heart Association Task Force on Practice Guidelines, and the American College of Physicians, American Association for Thoracic Surgery, Preventive Cardiovascular Nurses Association, Society for Cardiovascular Angiography and Interventions, and Society of Thoracic Surgeons. J Am Coll Cardiol. 2012; 60:e44-164.

[14] Grundy SM, Cleeman JI, Merz CN, Brewer HB Jr, Clark LT, Hunninghake DB, et al. Implications of recent clinical trials for the national cholesterol education program adult treatment panel III guidelines. Circulation. 2004; 110:227-39. Erratum in: Circulation. 2004; 110:763.

[15] Aronson D, Edelman ER. Coronary artery disease and diabetes mellitus. Cardiol Clin. 2014; 32:439-55.

[16] Jousilahti P, Vartiainen E, Tuomilehto J, Puska P. Sex, age, cardiovascular risk factors, and coronary heart disease: a prospective follow-up study of 14786 middle-aged men and women in Finland. Circulation. 1999; 99:1165-72.

[17] Niedziela J, Hudzik B, Niedziela N, Gąsior M, Gierlotka M, Wasilewski J, et al. The obesity paradox in acute coronary syndrome: a meta-analysis. Eur J Epidemiol. 2014; 29:801-12.

[18] Gruberg L, Weissman NJ, Waksman R, Fuchs S, Deible R, Pinnow $\mathrm{EE}$, et al. The impact of obesity on the short-term and long-term outcomes after percutaneous coronary intervention: the obesity paradox? J Am Coll Cardiol. 2002; 39:578-84.

[19] Sharma A, Lavie CJ, Borer JS, Vallakati A, Goel S, Lopez-Jimenez F, et al. Meta-analysis of the relation of body mass index to all-cause and cardiovascular mortality and hospitalization in patients with chronic heart failure. Am J Cardiol. 2015; 115:1428-34.

[20] Numasawa Y, Kohsaka S, Miyata H, Kawamura A, Noma S, Suzuki $\mathrm{M}$, et al. Impact of body mass index on in-hospital complications in patients undergoing percutaneous coronary intervention in a Japanese real-world multicenter registry. PLoS One. 2015; 10:e0124399. doi:10.1371/journal.pone.0124399.

[21] Neeland IJ, Kontos MC, de Lemos JA. Evolving considerations in the management of patients with left bundle branch block and suspected myocardial infarction. J Am Coll Cardiol. 2012; 60:96-105. 